\title{
Application of Copula-GARCH to Estimate VaR of a Portfolio with Credit Default Swaps
}

\author{
Jhe-Jheng Huang, Leh-Chyan So \\ Department of Quantitative Finance, National Tsing Hua University, Taiwan \\ Email: 1cso@mx.nthu.edu.tw
}

How to cite this paper: Huang, J.-J. and So, L.-C. (2018) Application of Copula-GARCH to Estimate VaR of a Portfolio with Credit Default Swaps. Journal of Mathematical Finance, 8, 382-407. https://doi.org/10.4236/jmf.2018.82025

Received: December 18, 2017

Accepted: May 15, 2018

Published: May 18, 2018

Copyright ( $) 2018$ by authors and Scientific Research Publishing Inc. This work is licensed under the Creative Commons Attribution International License (CC BY 4.0).

http://creativecommons.org/licenses/by/4.0/

(c) (i) Open Access

\begin{abstract}
Credit Default Swaps (CDSs) provide an efficient way for commercial banks to hedge their portfolios' exposure to credit risk. Following Patton (2006), Huang, Lee, Liang, and Lin (2009), and Fei, Fuertes, and Kalotychou (2013), we proposed a way to estimate Value-at-Risk (VaR) of portfolios containing CDSs that is better than the traditional methods mentioned in financial textbooks. Markit's North American Investment Grade CDX Index (CDX.NA.IG) is a combination index of 125 North American entities with investment-grade credit ratings that trade in the CDS market. Each of the S \& P 500 index and VIX are used with CDX.NA.IG to construct portfolios. This paper uses 2,477 daily data items from December 2004 to October 2014 covering the period of the subprime mortgage crisis and the European debt crisis. We chose six constant and two time-varying copula models combined with GARCH skewed Student-t innovation (GARCH-skt) to form eight copula-GARCH models to capture the joint distribution of the two assets in the portfolio. We then computed corresponding 1-day VaRs. According to our findings, the time-varying symmetrized Joe-Clayton (SJC) copula model combined with the GARCH-skt (tvSJC-copula-GARCH-skt) performed best, regardless of the market situation. Not surprisingly, this result stems mainly from this model's consideration of the serial correlation in the individual index return and the time-varying nonlinear dependency between indices.
\end{abstract}

\section{Keywords}

Copula, GARCH, VaR, CDX, VIX

\section{Introduction}

For commercial banks, credit risk arising from the defaults of their counterparties or borrowers is of great concern. Recently, credit derivatives have gained 
considerable popularity among the commercial banks as ways to diversify their exposure to credit risk. Among others, a credit default swap (CDS) is the most commonly used tool. Since its introduction in the mid-1990s, the trading volume of CDSs has been growing rapidly. According to Weistroffer, Speyer, and Walter (2009) [1], the peak occurred in 2007 with USD 58 trillion notional amounts outstanding, at over $70 \%$ of the debt securities outstanding worldwide at that time.

The protection seller of a CDS earns the premium by providing insurance to the protection buyer in case of a credit event due to the reference entity. In 2004, the CDS index was introduced to the market. The CDS index is a kind of standardized product providing more liquidity and efficiency than groups of single CDSs. Two well-known CDS indices are iTraxx and CDX. While iTraxx comprises 125 entities trading in the CDS market in Europe, Asia, the Middle East, and Africa, CDX consists of 125 North American companies. Our research object is Markit's North American Investment Grade CDX Index (CDX.NA.IG), an index containing 125 entities with investment-grade credit ratings involved in the North American CDS market.

A great deal of literature shows that CDSs have a connection with the stock index. Blanco, Bernnan, and Marsh (2005) [2] showed that CDSs with lower credit ratings could be more sensitive to stock returns than CDSs with higher ratings. Norden and Weber (2009) [3] claimed that during 2000-2002, the stock index return had a negative correlation with CDS return and with bond yield. Besides this, the negative correlation was more obviously observed in the CDS than in the bond yield. These authors' results imply that the CDS provides more information about stock index returns than bonds do, which corroborates the conclusions of Blanco et al. (2005) [2].

In addition to evidence for stock index returns, many researchers have provided evidence to show that CDSs have a great connection with the volatility index (e.g., Blanco et al., 2005 [2]; Ericsson, Jacobs, and Oviedo, 2009 [4]). By extending Merton's (1974) [5] model, Zhang, Zhou, and Zhu (2009) [6] found that equity volatility has convex, nonlinear effects on credit spreads. Making a comparison to an out-of-the-money put, Cao, Yu, and Zhong (2010) [7] claimed that the implied volatility deducted from the out-of-the-money put is an important determinant of the CDS spread.

Byström (2005) [8] showed that iTraxx Europe indices have significant autocorrelation positive coefficients, which implies that iTraxx indices are predictable and that the market is inefficient. In addition, iTraxx indices are negatively correlated to the stock index and positively to stock volatility. Through an OLS regression, Byström proposed that changes in iTraxx indices could mainly be explained by the stock index. Alexander and Kaeck (2008) [9] suggested that iTraxx Europe indices tend to commove more closely with stock volatility when the CDS market is extremely volatile; CDS spreads are more sensitive to stock returns than they are to stock volatility during stable periods. Fung, Sierra, Yau, 
and Zhang (2008) [10] provided evidence from the U.S. stock market and the CDS market to show that the volatility of both the investment-grade (CDX.NA.IG) and high-yield (CDX.NA.HY) CDS indices seems to lead the stock market volatility. However, the lead-lag relationship between these two markets depends on the credit rating of the reference entity. In particular, the high-yield CDS market is more closely related to the stock market than is the investment-grade CDS market. From the data of iTraxx Europe indices, European stock indices, and volatility indices during the period from 2004 to 2008, Giammarino and Barrieu (2009) [11] found that the dependence of these indices was not stable but involved structural changes.

Since many studies have shown that there is a certain way that the CDS market commoves with the stock market or the volatility, we are curious about the risk management issues of a CDS portfolio combining with a stock index or volatility index. Value at Risk $(\mathrm{VaR})$ is a commonly used measure of the market risk of an asset or a portfolio and describes the risk in an easy to understand way: in the next $\mathrm{N}$ days, we are $\mathrm{X} \%$ certain that the loss of the asset or the portfolio will not exceed the amount of VaR. Among the methods for estimating VaR suggested in the financial textbooks, most require that the joint distribution is known. It is impossible to get an accurate estimation for the calculation without consideration of the tail of distribution. While most of the above literature focuses on the linear correlation between CDS indices and stock index or volatility index, little of this literature has mentioned these indices' nonlinear or more complicated dependence structures. Hence, we apply the copula theory to allow construction of a flexible multivariate distribution with different margin distributions and different dependent structures, which relaxes the assumption of the normality and linear correlation of the portfolio's joint distribution.

Another important feature of financial data is the high heteroskedasticity, which means that volatility varies in time. The phenomenon is explained and fitted by the well-known GARCH model. The combination of the copula-GARCH model has often been mentioned in the discussion of computing VaR (e.g., Jondeau and Rockinger, 2006 [12]; Huang et al., 2009 [13]; Rong and Trück, 2010 [14]). By applying the copula-GARCH model to investigate the dependence structures among several European stock markets from 1980 to 1999, Jondeau and Rockinger (2006) [12] showed that their dependence becomes heavy when the market indices move in the same direction, regardless of whether they go up or down. Patton's (2006) [15] research on the dependence of the Deutsche mark and the yen claimed that these currencies' dependence is time-varying, and he constructed a flexible structure of the conditional dependence to model it. Huang et al. (2009) [13] used a copula-GARCH model to estimate the VaR of a portfolio consisting of the NASDAQ and TAIEX. By analyzing 2800 items of daily data covering the period of the subprime mortgage crisis in 2007-2009, So and $\mathrm{Yu}$ (2015) [16] showed that of the 16 copula-GARCH models used to depict the joint distribution of the two assets in the portfolio, the time-varying SJC copula 
model combined with the GARCH with Student-t innovation (tvSJC copula-GARCHt) was superior to the traditional VaR methods and performed best, regardless of the time period.

Since most of the above literature emphasizes the effects of different copula models, only the most commonly used GARCH $(1,1)$ with normal and Student-t innovations are taken into consideration. However, we cannot ignore the two prominent features often mentioned when it comes to financial volatilities: asymmetry and leverage (e.g., McAleer, 2014 [17]). Hansen (1994) [18] provided a GARCH model with a skewed Student-t density, which allows the margins to be of a negative skew and a fat-tailed distribution. In this study, we chose GARCH $(1,1)$ with residual terms following a skewed Student-t distribution to describe the marginal distribution of the asset returns, taking the negative skew, heteroskedasticity, and volatility clustering into consideration simultaneously.

The remainder of the paper is organized as follows. The methodology is presented in Section 2, the data are described in Section 3, the empirical results are analyzed in Section 4, and the conclusions are given in the final section.

\section{Methodology}

\subsection{Copula Model}

The term "copula" was originally mentioned in Sklar (1959) [19]. At the beginning, copula methods were applied in the field of statistics, but recently, these methods have gained considerable popularity in the field of finance. A copula is a kind of multidimensional probability distribution function that can be used to describe the dependency among several assets. In this study, we used a copula to discuss the CDS market's relationship with the stock market or with the volatility index; this is an application of a bivariate copula. A brief introduction of bivariate copulas follows.

\subsubsection{Sklar's Theorem}

Assume that the cumulative density functions of stochastic variables $X_{1}$ and $X_{2}$ are $F_{1}\left(x_{1}\right)=P\left(X_{1}<x_{1}\right)$ and $F_{2}\left(x_{2}\right)=P\left(X_{2}<x_{2}\right)$, respectively. After a process of probability integral transform, the cumulative density functions become the uniform distributions $u_{1}$ and $u_{2}$. The joint cumulative probability distribution of $X_{1}$ and $X_{2}$ can be represented by:

$$
\begin{aligned}
F\left(x_{1}, x_{2}\right) & =P\left(X_{1}<x_{1}, X_{2}<x_{2}\right) \\
& =C\left(F_{1}\left(x_{1}\right), F_{2}\left(x_{2}\right)\right)=C\left(u_{1}, u_{2}\right),
\end{aligned}
$$

where $C$ is called the copula function. Given that $F_{1}$ and $F_{2}$ are two continuous functions, there exists a certain copula function satisfying the above equation. On the other hand, given some specific copula function $C$ and cumulative distribution functions $F_{1}\left(x_{1}\right)$ and $F_{2}\left(x_{2}\right), \quad F\left(x_{1}, x_{2}\right)=C\left(F_{1}\left(x_{1}\right), F_{2}\left(x_{2}\right)\right)$ must be a two-dimensional cumulative distribution function with $F_{1}\left(x_{1}\right)$ and $F_{2}\left(x_{2}\right)$ as its marginals. 
The partial derivative of the above equation with respect to $x_{1}$ and $x_{2}$ is:

$$
\begin{aligned}
f\left(x_{1}, x_{2}\right) & =\frac{\partial^{2} F\left(x_{1}, x_{2}\right)}{\partial x_{1} \partial x_{2}}=\frac{\partial^{2} C\left(u_{1}, u_{2}\right)}{\partial u_{1} \partial u_{2}} \times \prod_{i=1}^{2} \frac{\partial F_{i}\left(x_{i}\right)}{\partial x_{i}} \\
& =c\left(u_{1}, u_{2}\right) \times f_{1}\left(x_{1}\right) \times f_{2}\left(x_{2}\right)
\end{aligned}
$$

The deduction gives us an idea that the joint density function consists of a copula and the two marginals; hence, we can set marginals and use the specific copula function to depict their relationship.

\subsubsection{Copula Functions}

The eight different types of copula functions used in this study are described as follows:

\section{Gaussian (Normal) copula}

$$
C_{\text {Gaussian }}\left(u_{1}, u_{2} ; \rho\right)=\Phi_{\rho}\left[\Phi^{-1}\left(u_{1}\right), \Phi^{-1}\left(u_{2}\right)\right],
$$

where $\Phi_{\rho}$ is the bivariate standardized Gaussian cumulative probability function, with the correlation $\rho$.

\section{Student-t copula}

$$
C_{T}\left(u_{1}, u_{2} ; \rho, v\right)=t_{\rho, v}\left[t_{v}^{-1}\left(u_{1}\right), t_{v}^{-1}\left(u_{2}\right)\right], \rho \in(-1,1),
$$

where $t_{\rho, v}$ is the bivariate Student-t cumulative probability function with the correlation $\rho$ and degree of freedom $v$. Similar to the Gaussian copula, the student- $t$ copula is symmetrically distributed. When the degree of freedom approaches infinite, the Student-t copula converges to the Gaussian copula. However, the Student- $t$ copula can capture the fat-tailed dependence feature of financial data.

Gumbel copula

$$
C_{\text {Gumbel }}\left(u_{1}, u_{2} ; \omega\right)=\exp \left(-\left(\left(-\log \left(u_{1}\right)\right)^{\omega}+\left(-\log \left(u_{2}\right)\right)^{\omega}\right)^{\frac{1}{\omega}}\right),
$$

where $\omega \in[1, \mathrm{e})$. The Gumbel (1960) [20] copula is an asymmetric function. When $u_{1}$ or $u_{2}$ approach 1 , the value of the Gumbel copula rises at a higher speed, which means that the dependence of these two variables becomes heavy when their values increase. Hence, the Gumbel copula can be used to capture the dependence around the tail of the right-hand side.

Clayton copula

$$
C_{\text {Clayton }}\left(u_{1}, u_{2} ; \delta\right)=\left(u_{1}^{-\delta}+u_{2}^{-\delta}-1\right)^{-\frac{1}{\delta}},
$$

where $\delta \in[-1, \infty)$. The Clayton (1978) [21] copula is also an asymmetric function. When $u_{1}$ or $u_{2}$ approach 0 , the value of the Clayton copula rises at a higher speed; that is, the dependence of these two variables becomes heavy when their values decrease. Hence, the Clayton copula, in contrast to the Gumbel copula, can be used to capture the dependence around the tail of the left-hand side.

Frank copula 


$$
C_{\text {Frank }}\left(u_{1}, u_{2} ; \tau\right)=-\frac{1}{\tau} \log \left(\frac{\tau\left(1-\mathrm{e}^{\tau}\right)-\left(1-\mathrm{e}^{\tau \cdot u_{1}}\right)\left(1-\mathrm{e}^{\tau \cdot u_{2}}\right)}{1-\mathrm{e}^{\tau}}\right),
$$

where $\tau \in(-\infty, 0) \cup(0, \mathrm{e})$. The Frank (1979) [22] copula is a symmetric function and is not suitable for depicting the dependence around the tails.

\section{Symmetrized Joe-Clayton (SJC) copula}

The SJC function is an expansion of the Joe-Clayton copula. Joe (1997) [23] proposed the Joe-Clayton copula:

$$
C_{J C}\left(u_{1}, u_{2} \mid \lambda^{U}, \lambda^{L}\right)=1-\left(1-\left(\left(1-\left(1-u_{1}\right)^{k}\right)^{-\gamma}+\left(1-\left(1-u_{2}\right)^{k}\right)^{-\gamma}-1\right)^{-\frac{1}{\gamma}}\right)^{\frac{1}{k}},
$$

where $k=\frac{1}{\log _{2}\left(2-\lambda^{U}\right)}, \quad \gamma=\frac{-1}{\log _{2}\left(2-\lambda^{L}\right)}, \quad \lambda^{U} \in(0,1), \quad \lambda^{L} \in(0,1) ; \quad \lambda^{U} \quad$ and $\lambda^{L}$, respectively, are the measures of the upper and lower tail dependence. A positive $\lambda^{U}$ or $\lambda^{L}$ implies that the copula has upper or lower tail dependence, while $\lambda^{U}=0$ means no upper tail dependence and $\lambda^{L}=0$ means no lower tail dependence. Upper and lower tail dependence count separately, and it is not necessary that these two features exist at the same time. It is notable

that the Joe-Clayton copula has the shortcoming that the pattern of this copula remains asymmetrical, even when the two tail dependence measures are equal. The SJC copula is a modification aiming to solve this problem. The SJC can guarantee symmetry when $\lambda^{U}=\lambda^{L}$.

The SJC copula is as follows:

$$
\begin{aligned}
& C_{\mathrm{SJC}}\left(u_{1}, u_{2} \mid \lambda^{U}, \lambda^{L}\right) \\
& =0.5 \cdot\left(C_{\mathrm{JC}}\left(u_{1}, u_{2} \mid \lambda^{U}, \lambda^{L}\right)+C_{J C}\left(1-u_{1}, 1-u_{2} \mid \lambda^{U}, \lambda^{L}\right)+u_{1}+u_{2}-1\right)
\end{aligned}
$$

where $\lambda^{U} \in(0,1), \lambda^{L} \in(0,1)$.

When the two variables are positively correlated, given $\alpha$, the upper tail dependence is denoted by:

$$
\lambda^{U}=\lim _{\alpha \rightarrow 1} \operatorname{Pr}\left(x_{2} \geq F_{2}^{-1}(\alpha) \mid x_{1} \geq F_{1}^{-1}(\alpha)\right) ;
$$

and the lower tail dependence is denoted by:

$$
\lambda^{L}=\lim _{\alpha \rightarrow 1} \operatorname{Pr}\left(x_{2} \leq F_{2}^{-1}(1-\alpha) \mid x_{1} \leq F_{1}^{-1}(1-\alpha)\right) .
$$

When the two variables are negatively correlated, given $\alpha$, the upper tail dependence is denoted by:

$$
\lambda^{U}=\lim _{\alpha \rightarrow 1} \operatorname{Pr}\left(x_{2} \geq F_{2}^{-1}(\alpha) \mid x_{1} \leq F_{1}^{-1}(1-\alpha)\right) ;
$$

and the lower tail dependence is denoted by:

$$
\lambda^{L}=\lim _{\alpha \rightarrow 1} \operatorname{Pr}\left(x_{2} \leq F_{2}^{-1}(1-\alpha) \mid x_{1} \geq F_{1}^{-1}(\alpha)\right) .
$$

It should be noted that in this study, since CDX.NA.IG and S \& P500 are negatively correlated, S \& P500 is denoted by $x_{1}$ and CDX.NA.IG by $x_{2}$. 
When $\lambda^{U}$ or $\lambda^{L}$ approach 1, the upper or lower tail dependence is higher. In contrast, when $\lambda^{U}$ or $\lambda^{L}$ equals 0 , there is no tail dependence. There is no necessary relationship between the two parameters.

Time-varying Symmetrized Joy-Clayton (Time-varying SJC) copula

The copula functions mentioned above all belong to static models, which means that the dependence between two assets are assumed to remain unchanged over time or market situations. However, the realistic case observed in the CDS and stock markets is that their dependence varies across time (e.g., Alexander and Kaeck, 2008 [9]; Fung et al., 2008 [10]; Giammarino and Barrieu, 2009 [11]). Therefore, we introduce time-varying copulas to capture this feature.

According to Patton (2006) [15], the time-varying SJC copula and time-varying Gaussian (Normal) copula keep their same original functional form with time-varying dependence parameters following an ARMA $(1,10)$ evolution process.

The time-varying SJC copula is as follows:

$$
\begin{gathered}
C_{t-\mathrm{SJC}}\left(u_{1}, u_{2} \mid \tau_{t}^{U}, \tau_{t}^{L}\right) \\
=0.5 \cdot\left(C_{\mathrm{JC}}\left(u_{1}, u_{2} \mid \tau_{t}^{U}, \tau_{t}^{L}\right)+C_{J C}\left(1-u_{1}, 1-u_{2} \mid \tau_{t}^{U}, \tau_{t}^{L}\right)+u_{1}+u_{2}-1\right) \\
\tau_{t}^{U}=\Lambda\left(\omega_{U}+\beta_{U} \tau_{t-1}^{U}+\alpha_{U} \cdot \frac{1}{10} \sum_{j=1}^{10}\left|u_{1, t-j}-u_{2, t-j}\right|\right), \\
\tau_{t}^{L}=\Lambda\left(\omega_{L}+\beta_{L} \tau_{t-1}^{L}+\alpha_{L} \cdot \frac{1}{10} \sum_{j=1}^{10}\left|u_{1, t-j}-u_{2, t-j}\right|\right),
\end{gathered}
$$

where $\Lambda=\left(1+\mathrm{e}^{-x}\right)^{-1}, \tau_{t}^{U} \in(0,1), \tau_{t}^{L} \in(0,1) ; \Lambda$ is a logistic transformation to ensure that $\tau_{t}^{U}$ and $\tau_{t}^{L}$ are in the range of $(0,1)$.

Time-varying normal copula

$$
\begin{gathered}
C_{t-\text { Normal }}\left(u_{1}, u_{2} ; \rho_{t}\right)=\Phi\left(\Phi^{-1}\left(u_{1}\right), \Phi^{-1}\left(u_{2}\right)\right), \\
\rho_{t}=\tilde{\Lambda}\left(\omega_{\rho}+\beta_{\rho} \cdot \rho_{t-1}+\alpha \cdot \frac{1}{10} \sum_{j=1}^{10} \Phi^{-1}\left(u_{1, t-j}\right) \cdot \Phi^{-1}\left(u_{2, t-j}\right)\right),
\end{gathered}
$$

where $\tilde{\Lambda}(x)=\left(1-\mathrm{e}^{-x}\right)\left(1+\mathrm{e}^{-x}\right)^{-1}=\tanh \left(\frac{x}{2}\right), \quad \rho_{t} \in(-1,1) ; \tilde{\Lambda}(x)$ is a logistic transformation to ensure that $\rho_{t}$ is in the range of $(-1,1)$.

\subsection{Marginal Distribution}

Considerable empirical evidence shows that asset returns do not necessarily follow normal distribution. In most cases, they are negatively skewed or fat-tailed. Longin and Solink (2001) [24] and Okimoto (2008) [25] showed that the dependence among international equity markets becomes heavy when markets are bearish rather than bullish. The evidence of Ang and Chen (2002) [26] from the data of stock markets in the United States also echoed the findings. As for the CDS index, Fei, Fuertes and Kalotychou (2013) [27] investigated the dependence between iTraxx or its subindices and the European stock index or volatility index 
from 2005 to 2011 . They found that the return processes tend to be negatively skewed. In addition, when markets are in an extremely bad situation, their dependence becomes heavier.

The other well-known feature frequently cited in the financial literature is the high heteroskedasticity, which means that the return volatility varies in time. The GARCH model is the most common tool used to capture this feature observed in financial data.

We chose GARCH $(1,1)$ with residual terms following a skewed Student-t distribution to describe the marginal distribution of the asset returns, simultaneously taking the negative skew, heteroskedasticity, and volatility clustering into consideration.

The skewed Student-t distribution, proposed by Hansen (1994) [18], is described as follows:

$$
d(x ; \eta ; \lambda)=\left\{\begin{array}{l}
b c\left(1+\frac{1}{\eta-2}\left(\frac{b x+a}{1-\lambda}\right)^{2}\right)^{-\left[\frac{\eta+1}{2}\right]}, \text { if } x<-\frac{a}{b} \\
b c\left(1+\frac{1}{\eta-2}\left(\frac{b x+a}{1+\lambda}\right)^{2}\right)^{-\left[\frac{\eta+1}{2}\right]}, \text { if } x \geq-\frac{a}{b}
\end{array}\right.
$$

where $a \equiv 4 \lambda c \frac{\eta-2}{\eta-1}, b^{2} \equiv 1+3 \lambda^{2}-a^{2}, c \equiv \frac{\Gamma\left(\frac{\eta+1}{2}\right)}{\sqrt{\pi(\eta-2)} \Gamma\left(\frac{\eta}{2}\right)}$.

The variables $\eta$ and $\lambda$ represent the degree of freedom and the measure of asymmetry, respectively. If $\lambda=0$, the distribution degenerates to the Student- $t$ distribution. If $\eta$ approaches infinity at the same time, the distribution degenerates to the normal distribution.

Denote the probability distribution for $x, d(x ; \eta ; \lambda)$, by $x \sim s k t(\eta, \lambda)$. The $\operatorname{GARCH}(1,1)$ with a skewed Student-t distribution is as follows:

$$
\begin{gathered}
x_{t}=\mu+e_{t}, \\
e_{t}=\varepsilon_{t} \sigma_{t}, \\
\sigma_{t}^{2}=\alpha_{0}+\alpha_{1} e_{t-1}^{2}+\beta \sigma_{t-1}^{2}, \\
\varepsilon_{t} \sim \operatorname{skt}(\eta, \lambda),
\end{gathered}
$$

where $\mu=E\left(x_{t}\right)=E\left(E\left(x_{t} \mid \Omega_{t-1}\right)\right)=E\left(\mu_{t}\right)=\mu$ is the unconditional mean, $\sigma_{t}^{2}=\operatorname{Var}\left(x_{t} \mid \Omega_{t-1}\right)$ is the conditional variance, and $\Omega_{t-1}$ is all the information obtained at $t-1$.

\subsection{Parameter Estimation}

When applying the maximum likelihood method, we need to estimate the parameters in the copula function and the GARCH model at the same time. However, the computation process is time-wasting when parameters are abundant. Hence, we adopted the two-stage inference function for margins (IFMs) method proposed 
by Joe (2005) [23] to estimate the GARCH and copula parameters separately. The estimates using the IFM method share the features of consistency and asymptotic normality.

In the first stage, the log-likelihood function of the two marginal distributions should be considered:

$$
\begin{gathered}
\mathcal{L}_{i}\left(\theta_{i}\right)=\sum_{t=1}^{T} \sum_{i=1}^{2} \ln f_{i}\left(x_{i t} ; \theta_{i}\right), i=1,2, \\
\hat{\theta}_{i, M L E}=\arg \max \mathcal{L}_{i}\left(\theta_{i}\right), i=1,2 .
\end{gathered}
$$

In the second stage, we have to substitute the estimates obtained in the first stage into the following equation and estimate the copula parameters:

$$
\begin{gathered}
\mathcal{L}(\varphi)=\sum_{t=1}^{T} \ln c\left(F_{1}\left(x_{1 t} ; \widehat{\theta}_{1}\right), F_{2}\left(x_{2 t}, \widehat{\theta_{2}}\right) ; \varphi\right), \\
\hat{\varphi}_{i, M L E}=\arg \max \mathcal{L}(\varphi)
\end{gathered}
$$

\subsection{Value at Risk (VaR)}

Value at Risk expresses the idea of the greatest potential loss, given a certain confidence level, over a certain time period. By definition, given the confidence level $\alpha$, the 1-day VaR is:

$$
P\left(R_{t+1} \leq \operatorname{VaR}_{\alpha} \mid \Omega_{t}\right)=\alpha,
$$

where $R_{t+1}$ is the asset return, and $\Omega_{t}$ is all the information obtained at time $t$. This equation explains that we are $100 \times(1-\alpha) \%$ certain that the loss of the asset or the portfolio in the next day will not exceed the amount of $V a R_{\alpha} ; \alpha$ is usually set at $5 \%$ or $1 \%$. The lower the value of $\alpha$, the higher the VaR. The 1 -day VaR can be extended to a T-day VaR by:

$$
\operatorname{VaR}(T \text { days })=\operatorname{VaR}_{\alpha} \cdot \sqrt{T}
$$

where $\operatorname{VaR}_{\alpha}$ is the 1-day VaR.

To make comparisons, we chose four estimation methods typically suggested in the financial textbooks (e.g., Hull (2012) [28]) to compute the value of $\mathrm{VaR}$ - the historical simulation, the variance-covariance method, exponential weighted, and univariate GARCH. At the end of this subsection, we describe the copula-GARCH model highlighted in this study in more detail.

\subsubsection{Historical Simulation}

Reorder the daily return data over the last $T$ days, $x_{p, t}, t=1,2, \cdots, T$, from low to high. The $[T \times \alpha]^{\text {th }}$ worst value on the reordered list is the required $\operatorname{VaR}_{\alpha}$. The assumption behind the historical simulation is that the future repeats the past. However, when some rare event happens, a historical simulation tends to underestimate the risk.

\subsubsection{Variance-Covariance Method}

The variance and covariance method assumes that the returns are all normally 
distributed.

The $t+1$ 1-day VaR can be represented as follows:

$$
\operatorname{VaR}_{t+1}(\alpha)=\mu_{p, t+1}+\sigma_{p, t+1} \cdot \Phi^{-1}(\alpha),
$$

where $\sigma_{p, t+1}^{2}=\left[\begin{array}{ll}w_{1} & w_{2}\end{array}\right] \cdot\left[\begin{array}{ll}\sigma_{11, t+1} & \sigma_{12, t+1} \\ \sigma_{21, t+1} & \sigma_{22, t+1}\end{array}\right] \cdot\left[\begin{array}{l}w_{1} \\ w_{2}\end{array}\right]=w \Sigma_{t+1} w^{\prime}$,

$$
\sigma_{i j, t+1}^{2}=\frac{1}{t-1} \sum_{h=1}^{t}\left(x_{i h}-\overline{x_{i}}\right)\left(x_{j h}-\overline{x_{j}}\right) ; i=1,2 ; j=1,2 \text {, }
$$

$\mu_{p, t+1}$ and $\sigma_{p, t+1}$ are the mean and standard deviation of the portfolio return on day $t+1 ; w_{i}$ is the portfolio weight; and $\Phi^{-1}(\alpha)$ is the inverse function of the cumulative distribution function of the standard normal.

\subsubsection{Exponentially Weighted Moving Average (EWMA)}

This method also assumes that the returns are all normally distributed. Different from the variance and covariance method, EWMA puts a relatively heavy weight on the most recent data. The $t+11$-day VaR can be represented as follows:

$$
\operatorname{VaR}_{t+1}(\alpha)=\mu_{p, t+1}+\sigma_{p, t+1} \cdot \Phi^{-1}(\alpha),
$$

where $\sigma_{p, t+1}^{2}=\left[\begin{array}{ll}w_{1} & w_{2}\end{array}\right] \cdot\left[\begin{array}{ll}\sigma_{11, t+1} & \sigma_{12, t+1} \\ \sigma_{21, t+1} & \sigma_{22, t+1}\end{array}\right] \cdot\left[\begin{array}{l}w_{1} \\ w_{2}\end{array}\right]=w \Sigma_{t+1} w^{\prime}$,

$$
\sigma_{i j, t+1}^{2}=\lambda \cdot \sigma_{i j, t}^{2}+(1-\lambda) \cdot u_{i j, t}^{2} ; i=1,2 ; j=1,2 ; 0 \leq \lambda \leq 1 \text {, }
$$

$\mu_{p, t+1}$ and $\sigma_{p, t+1}$ are the mean and standard deviation of the portfolio return on day $t+1 ; \quad w_{i}$ is the portfolio weight; and $\Phi^{-1}(\alpha)$ is the inverse function of the cumulative distribution function of the standard normal.

\subsubsection{Univariate GARCH-VaR Method}

The GARCH $(1,1)$ with a skewed Student-t distribution is used to fit the in-sample portfolio return:

$$
\begin{gathered}
x_{p, t}=\mu+e_{t}, \\
e_{t}=\varepsilon_{t} \sigma_{p, t}, \\
\sigma_{p, t}^{2}=\alpha_{0}+\alpha_{1} e_{t-1}^{2}+\beta \sigma_{p, t-1}^{2}, \\
\varepsilon_{t} \sim \operatorname{skt}(\eta, \lambda) ;
\end{gathered}
$$

where $\mu=E\left(x_{p, t}\right)=E\left(E\left(x_{p, t} \mid \Omega_{t-1}\right)\right)=E\left(\mu_{t}\right)=\mu$ is the unconditional mean, $\sigma_{t}^{2}=\operatorname{Var}\left(x_{p, t} \mid \Omega_{t-1}\right)$ is the conditional variance, and $\Omega_{t-1}$ is all the information obtained at $t-1$.

We then estimate the out-of-the-sample conditional volatility of the portfolio return. The $t+11$-day VaR can easily be obtained by:

$$
\operatorname{VaR}_{p, t+1}(\alpha)\left|\Omega_{t}=\left(x_{p, t+1}+\sigma_{p, t+1} \cdot \Psi^{-1}(\alpha)\right)\right| \Omega_{t} ;
$$

where $x_{p, t+1}$ and $\sigma_{p, t+1}$ are the mean and standard deviation of the portfolio return on day $t+1$, as estimated on day $t$, and $\Psi^{-1}(\alpha)$ is the inverse function 
of the cumulative distribution function of a skewed Student-t.

\subsubsection{Copula-GARCH Method}

For each portfolio, we collected the two indices' returns on the last $T$ days to estimate their joint distribution on the $T+1$ day and the 1-day VaR, $\operatorname{VaR}_{T+1}$. The procedure is described as follows:

1) Use the GARCH with skewed Student-t (GARCH-skt) model to fit the T in-sample data and obtain the standardized residuals and returns of these two asset returns.

2) Apply the probability integral transform to each standardized return and obtain a uniformly distributed random vector $\left\{\left(u_{1 n}, u_{2 n}\right)\right\}_{n=1}^{n=T}$.

3) Estimate the copula parameters of $\left\{\left(u_{1 n}, u_{2 n}\right)\right\}_{n=1}^{n=T}$ by using the maximum likelihood method. Substitute the estimated parameters into copulas to get uniformly distributed random vectors $\left\{\left(S_{1 n}, S_{2 n}\right)\right\}_{n=1}^{n=N}$, each with $N$ elements. Note that $\left\{\left(S_{1 n}, S_{2 n}\right)\right\}_{n=1}^{n=N}$ contains all information about the dependence structure between the two assets.

4) Apply the probability integral transform to $\left\{\left(S_{1 n}, S_{2 n}\right)\right\}_{n=1}^{n=N}$, and substitute the unconditional means and conditional variances of the GARCH models to obtain a set of simulated asset returns $\left\{\left(R_{1 n}, R_{2 n}\right)\right\}_{n=1}^{n=N}$.

5) Assume that the portfolio return is calculated by the equally weighted average of the two asset returns. Use the results of step 4 to calculate the $\operatorname{VaR}_{T+1}$ of the portfolio return at $95 \%$ and $99 \%$.

6) Apply the rolling window method to do backtesting. When a new observation is added to the data, remove the oldest one. Repeat the above five steps $M$ times to obtain $M$ out-sample 1-day VaRs.

\section{Data}

In this study, we focused on the CDS market. We investigated its dependent structure with respect to the stock index or volatility index, and then we estimated the VaRs of the portfolio containing the CDS index first with the stock index and then with the volatility index.

We collected the data of S \& P500, VIX, and the Markit's North American Investment Grade CDX Index (CDX.NA.IG), which is a five-year CDS index with the highest market liquidity. We obtained the data from the Bloomberg Database, which provides accurate high-quality cross-asset, real-time and historical time-series data in all markets including equity, fixed income and FX. From each index, we collected 2477 daily adjusted closing prices from December 1, 2004, to October 31, 2014. Of the 2477 samples, the first 550 daily prices were used as in-sample data. For the purpose of comparison, the rest of the samples were divided into three out-sample periods for backtesting. The first 651 days from February 27, 2007, to October 2, 2009, we named the subprime crisis period, which began from the date that Freddie Mac announced that it would no longer purchase the most risky subprime mortgages and mortgage-related securities; ${ }^{1}$ Refer to: https://www.stlouisfed.org/financial-crisis/full-timeline. 
the second 601 days from October 5, 2009, to February 10, 2012, we considered the European debt crisis period, beginning from the date that the Greek government revealed that its budget deficit had risen to an extremely high level of its GDP, resulting in market panic; ${ }^{2}$ the last 675 days from February 13, 2012, to October 31, 2014, we named the steady period, beginning from the date that the Greek government won parliamentary approval for austerity measures to secure an international bailout. ${ }^{3}$

The daily return of the index is computed by:

$$
r_{t}=\ln \frac{P_{t}}{P_{t-1}},
$$

where $P_{t}$ refers to the adjusted closing price at time $t$.

\section{Empirical Results}

In Table 1, we see that the skewnesses of all three indices are not equal to zero, and their kurtoses are larger than 3 , which shows that the data share the features of a fat tail and negative skew. Using the Jarque-Bera test, we found that the index returns do not follow a normal distribution. Figure 1 shows the feature of volatility clustering, dramatic movements tend to be followed by dramatic movements. All three index returns changed the most dramatically during the subprime crisis period and the most modestly during the steady period. Among the three, the VIX return was the most volatile.

After using GARCH $(1,1)$ with a skewed Student-t distribution (GARCH-skt) to fit the marginal distributions of the two index returns, we applied the probability integral transform to each standardized return and obtained a set of

Table 1. Descriptive statistics of index returns.

\begin{tabular}{cccc}
\hline Descriptive statistics & CDX.NA.IG & S \& P500 & VIX \\
\hline Observations & 2477 & 2477 & 2477 \\
Mean & 0.000134 & 0.000213 & 0.0000317 \\
Standard Deviation & 0.030972 & 0.012949 & 0.06919 \\
Skewness & 0.337114 & -0.48688 & 0.714619 \\
Kurtosis & 9.245011 & 13.95537 & 6.998307 \\
Jarque-Bera test & 4072.002 & 12484.81 & 1860.507 \\
& $(0.00000)$ & $(0.00000)$ & $(0.00000)$ \\
\hline
\end{tabular}

We collected the data of the Markit's North American Investment Grade CDX Index (CDX.NA.IG), S \& P500, and VIX. From each index, we collected 2477 daily adjusted closing prices from December 1, 2004, to October 31, 2014. The results of the Jarque-Bera test to detect if the returns follow a normal distribution are also presented.

\footnotetext{
${ }^{2}$ Refer to:

http://www.bloomberg.com/news/articles/2010-10-06/greece-s-debt-deficit-figures-for-2006-2009-t o-be-revised-upward-eu-says

${ }^{3}$ Refer to:

http://www.bloomberg.com/news/articles/2012-02-12/papademos-wins-sufficient-support-in-parlia ment-for-greek-austerity-plans
} 

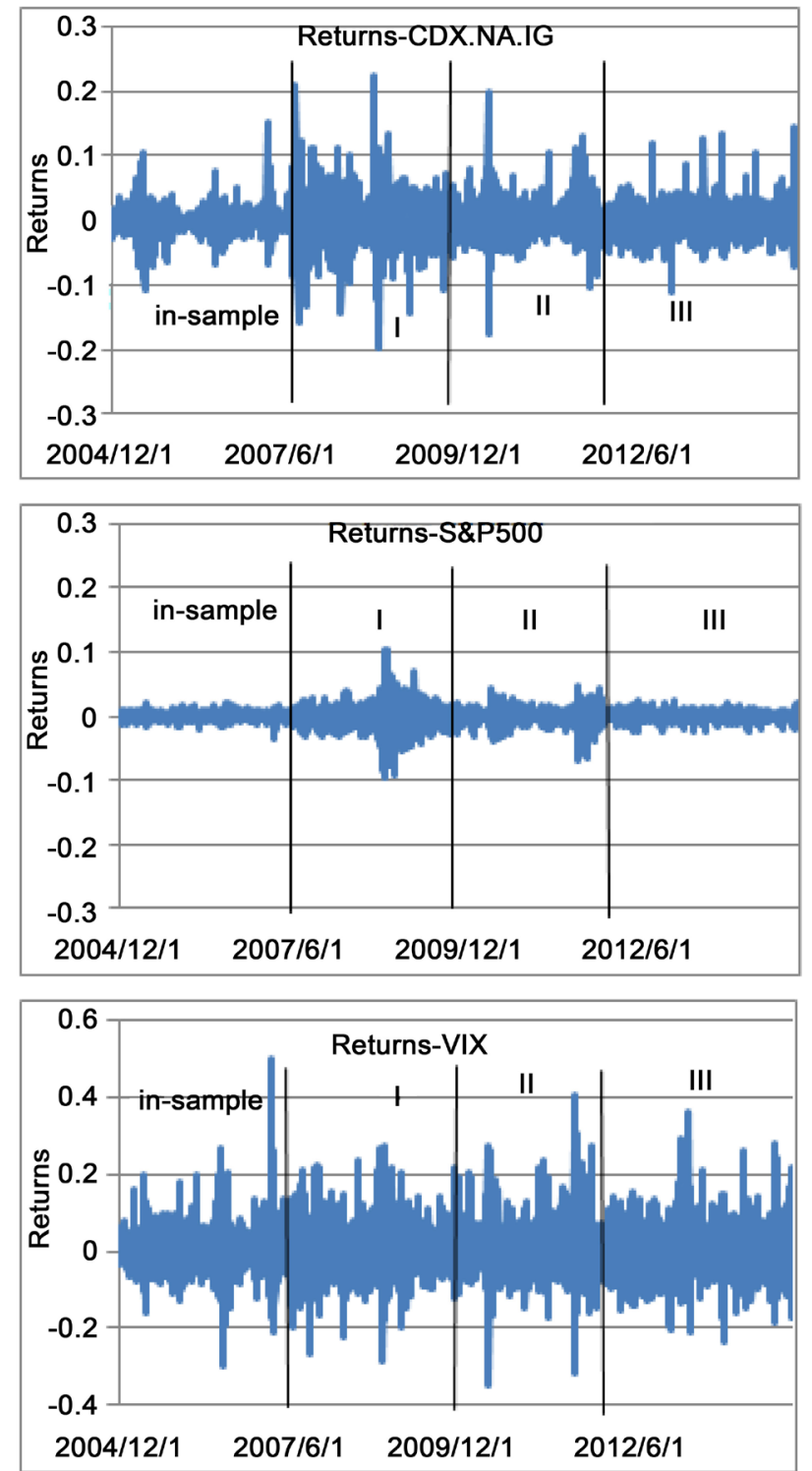

This figure shows the patterns of the three index returns during different sub-periods: the in-sample period, the subprime crisis period, the European debt crisis period, and the steady period.

Figure 1. Daily returns of indices.

uniformly distributed random vectors. We then estimated the copula parameters and constructed the copula-GARCH models. We chose the copula models that were the best fitted in terms of AIC statistic.

\subsection{Marginal Distributions}

Table 2 shows the results of the parameters estimated by the GARCH-skt model. The estimated parameters are all significantly different from zero. We used the Kolmogorov-Smirnov goodness-of-fit test (K-S test) to check that after the probability integral transform, the standardized residuals followed a uniform distribution $(0,1)$. In addition, we applied the Ljung-Box Q-test and $\mathrm{Q}^{2}$-test to the lag- $1,-3$, and -5 terms of the standardized residuals, and we cannot reject the 
Table 2. Results for GARCH-skt.

\begin{tabular}{|c|c|c|c|}
\hline Parameters & CDX.NA.IG & S \& P500 & VIX \\
\hline$\mu$ & $\begin{array}{c}-0.001217 \\
(0.0000)\end{array}$ & $\begin{array}{c}0.000649 \\
(0.0000)\end{array}$ & $\begin{array}{l}0.00007 \\
(0.0000)\end{array}$ \\
\hline$a_{0}$ & $\begin{array}{c}0.000019 \\
(0.0000)\end{array}$ & $\begin{array}{c}0.000001 \\
(0.0000)\end{array}$ & $\begin{array}{r}0.000435 \\
(0.0034)\end{array}$ \\
\hline$\alpha_{1}$ & $\begin{array}{c}0.126910 \\
(0.0000)\end{array}$ & $\begin{array}{c}0.100361 \\
(0.0012)\end{array}$ & $\begin{array}{c}0.170195 \\
(0.0001)\end{array}$ \\
\hline$\beta$ & $\begin{array}{c}0.860450 \\
(0.0000)\end{array}$ & $\begin{array}{c}0.887245 \\
(0.0000)\end{array}$ & $\begin{array}{c}0.744291 \\
(0.0000)\end{array}$ \\
\hline$\eta$ & $\begin{array}{c}2.0006 \\
(0.0000)\end{array}$ & $\begin{array}{c}3.9225 \\
(0.0001)\end{array}$ & $\begin{array}{c}3.6960 \\
(0.0000)\end{array}$ \\
\hline$\lambda$ & $\begin{array}{c}0.0956 \\
(0.0000)\end{array}$ & $\begin{array}{l}-0.0651 \\
(0.0000)\end{array}$ & $\begin{array}{c}0.1580 \\
(0.0000)\end{array}$ \\
\hline Q-test (1) & $(0.1954)$ & $(0.1128)$ & $(0.0665)$ \\
\hline Q-test (3) & $(0.1623)$ & $(0.1654)$ & $(0.1420)$ \\
\hline Q-test (5) & $(0.2158)$ & $(0.2067)$ & $(0.2689)$ \\
\hline $\mathrm{Q}^{2}$-test $(1)$ & $(0.0986)$ & $(0.0863)$ & $(0.1642)$ \\
\hline $\mathrm{Q}^{2}$-test (3) & $(0.1247)$ & $(0.1346)$ & $(0.1369)$ \\
\hline$Q^{2}$-test (5) & $(0.2985)$ & $(0.1651)$ & $(0.3436)$ \\
\hline $\mathrm{K}-\mathrm{S}$ test & $(0.3779)$ & $(0.2065)$ & $(0.6842)$ \\
\hline
\end{tabular}

Of the 2477 samples, the first 550 daily prices were used as in-sample data. We used the GARCH $(1,1)$ with skewed Student-t (GARCH-skt) model to fit the in-sample indices' returns. This table reports the results of the parameters estimated by the GARCH $(1,1)$ with a skewed Student-t distribution:

$$
x_{t}=\mu+e_{t}, e_{t}=\varepsilon_{t} \sigma_{t}, \sigma_{t}^{2}=\alpha_{0}+\alpha_{1} e_{t-1}^{2}+\beta \sigma_{t-1}^{2}, \varepsilon_{t} \sim s k t(\eta, \lambda),
$$

We say that $x \sim \operatorname{skt}(\eta, \lambda)$ means that the probability distribution for $x, d(x ; \eta ; \lambda)$ follows:

$$
\begin{aligned}
& d(x ; \eta ; \lambda)= \begin{cases}b c\left(1+\frac{1}{\eta-2}\left(\frac{b x+a}{1-\lambda}\right)^{2}\right)^{-\left[\frac{\eta+1}{2}\right]}, \text { if } x<-\frac{a}{b} \\
b c\left(1+\frac{1}{\eta-2}\left(\frac{b x+a}{1+\lambda}\right)^{2}\right)^{-\left[\frac{\eta+1}{2}\right]}, \text { if } x \geq-\frac{a}{b}\end{cases} \\
& \text { where } a \equiv 4 \lambda c \frac{\eta-2}{\eta-1}, b^{2} \equiv 1+3 \lambda^{2}-a^{2}, c \equiv \frac{\Gamma\left(\frac{\eta+1}{2}\right)}{\sqrt{\pi(\eta-2)} \Gamma\left(\frac{\eta}{2}\right)} \text {. }
\end{aligned}
$$

The results of the Ljung-Box Q-test, $\mathrm{Q}^{2}$-test, and the Kolmogorov-Smirnov goodness-of-fit test (K-S test) are also presented. The $\mathrm{p}$-values are in parentheses.

null hypothesis that the standardized residuals followed a uniform distribution $(0,1)$ and there is no autocorrelation. The above tests guaranteed that we could estimate parameters in copulas accurately.

\subsection{Copulas}

The parameter estimates of the eight copulas are shown in Table 3. From the estimated Gaussian copula parameters, we found that the absolute value of asset 
Table 3. Results for copula-GARCH models.

\begin{tabular}{cccc}
\hline copula & parameter & CDX.NA.IG S \& P500 & CDX.NA.IG VIX \\
\hline Gaussian & $\rho$ & -0.6952 & 0.6248 \\
Student-t & $\rho$ & -0.7212 & 0.6375 \\
Clayton & $v$ & 4.2414 & 6.7759 \\
Gumbel & $\delta$ & 1.3322 & 0.9711 \\
Frank & $\omega$ & 1.9815 & 1.7395 \\
SJC & $\tau$ & -6.2039 & 4.7747 \\
& $\lambda^{U}$ & 0.4808 & 0.5013 \\
& $\lambda^{\prime}$ & 0.5525 & 0.3456 \\
& $\omega_{U}$ & -1.7002 & 1.0137 \\
Time-varying SJC & $\alpha_{U}$ & -1.4526 & -3.1406 \\
& $\beta_{U}$ & 3.8828 & -0.8633 \\
& $\omega_{L}$ & -1.9516 & -1.9341 \\
& $\alpha_{L}$ & -0.3817 & -0.5891 \\
& $\beta_{L}$ & 4.0453 & 4.0637 \\
Time-varying nor- & $\omega_{\rho}$ & 4.1369 & 3.4916 \\
& $\alpha_{\rho}$ & -0.7603 & -0.2037 \\
& $\beta_{\rho}$ & -2.4695 & -3.0003 \\
\hline
\end{tabular}

Each of the S \& P 500 index and VIX are used with CDX.NA.IG to construct portfolios. This table shows the parameter estimates of the eight copulas for the two portfolios. Of the 2477 samples, the first 550 daily prices were used as in-sample data. After fitting the in-sample indices' returns with the GARCH-skt model, we applied the probability integral transform to each standardized return and obtained a set of uniformly distributed random vectors. We then estimated the copula parameters for each of the portfolios.

returns is about $0.6-0.7$. The correlation coefficient of CDX.NA.IG with S \& P500 is negative, while that with VIX is positive. The results correspond to the findings reported in Byström (2005) [8] and Norden and Weber (2009) [3]. From the SJC copula parameters, we found that the dependence structure between asset returns could be asymmetrical; in particular, the lower tail dependence is higher than the upper tail in the case of the portfolio containing CDX.NA.IG and S \& P500. By definition, this implies that the probability of CDX.NA.IG dropping given that S \& $\mathrm{P} 500$ rises is higher than that of CDX.NA.IG increasing given that $S \&$ P500 decreases. In contrast, the upper tail dependence is higher than the lower tail in the case of the portfolio containing CDX.NA.IG and VIX, which implies that the probability of CDX.NA.IG increasing with VIX is higher than that of CDX.NA.IG dropping with VIX. This result corresponds to Alexander and Kaeck's (2008) [9] findings, which showed that when markets are bullish, the sensitivity of the CDS index to S \& P500 is much stronger than to VIX, and when markets are bearish, the sensitivity of the CDS index to VIX is much stronger than to S \& P500.

AIC statistic was used to find the best copula-GARCH model, shown in Table 4. According to AIC, we found that the dynamic normal copula and the dynamic 
Table 4. AIC for copula-GARCH.

\begin{tabular}{ccc}
\hline copula & CDX.NA.IG S \& P500 & CDX.NA.IG VIX \\
\hline Gaussian & -0.6594 & -0.4941 \\
Student-t & -0.7381 & -0.5245 \\
Clayton & -0.5791 & -0.3402 \\
Gumbel & -0.6544 & -0.5118 \\
Frank & -0.6687 & -0.4616 \\
SJC & -0.6974 & -0.5190 \\
Time-varying SJC & -0.8030 & -0.5302 \\
Time-varying normal & -0.7078 & -0.4932 \\
\hline
\end{tabular}

This Table shows the results of AIC statistic for our copula-GARCH models. We substituted the estimated parameters into copulas to get uniformly distributed random vectors and applied the probability integral transform to the vectors. We then substituted the unconditional means and conditional variances of the GARCH models to obtain a set of simulated asset returns. We chose the copula models that were the best fitted in terms of AIC statistic. For each portfolio, the lowest AIC among the eight copulas is marked in bold.

SJC copula were relatively more adequate copulas for describing data than were the static normal copula and static SJC copula. Since dynamic copulas can capture time-varying relations between two index returns, they provide up-to-date adjustments for political or financially rare events, which is an advantage of the dynamic copulas over static ones. The best suitable model was the time-varying SJC. Hence, we claim that the assumption of the GARCH-skt model combined with the time-varying SJC copula, tvSJC-copula-GARCH-skt, had the best goodness-of-fit to the portfolio consisting of CDX.NA.IG. This finding is similar to that of So and $\mathrm{Yu}$ (2015) [16], who provided evidence to show that the GARCH model combined with time-varying SJC offered the best goodness-of-fit to the portfolio containing U.S. REITs.

\subsection{Analysis of the tvSJC-Copula-GARCH-skt}

Figure 2 and Figure 3 illustrate the upper and lower tail dependences of CDX.NA.IG and the other index returns. For the portfolio containing CDX.NA.IG and $S \&$ P500, the lower tail dependence is higher than the upper tail dependence, which implies that the probability of CDX.NA.IG decreasing as S \& P500 increases is higher than that of CDX.NA.IG increasing as S \& P500 decreases. In contrast, the upper tail dependence is higher than the lower tail in the case of the portfolio containing CDX.NA.IG and VIX, which implies that the probability of CDX.NA.IG increasing with VIX is higher than that of CDX.NA.IG decreasing with VIX. There is no difference from the case of the static SJC copula, which showed that when markets are bullish, the sensitivity of the CDS index to S \& P500 is much stronger than to VIX, and when markets are bearish, the sensitivity of the CDS index to VIX is much stronger than to S \& P500. As for the case of CDX.NA.IG and S \& P500, we also observed that both of the upper and lower tail dependences are at a relatively high level around September 2008 when the 
market suffered the most severe impact due to the subprime crisis.

\subsection{Backtesting of VaR using tvSJC-Copula-GARCH-skt and Other Traditional Methods}

The traditional methods referred in this study are historical simulation, the variance-covariance method, EWMA, and the univariate GARCH-skt method. As mentioned previously, in order to calculate a 1-day VaR, we collected the index returns on the last $\mathrm{T}$ days to estimate their joint distribution on the $\mathrm{T}+1$ day

Upper tail dependence: CDX.NA.IG \& S\&P500

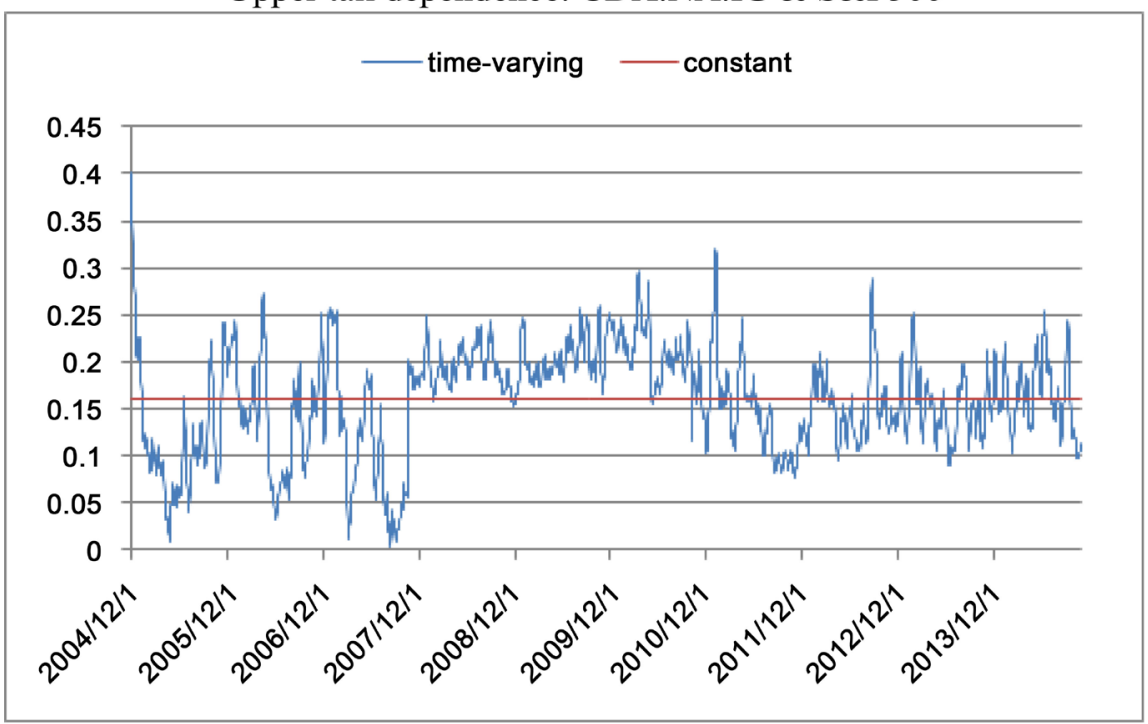

Lower tail dependence: CDX.NA.IG \& S\&P500

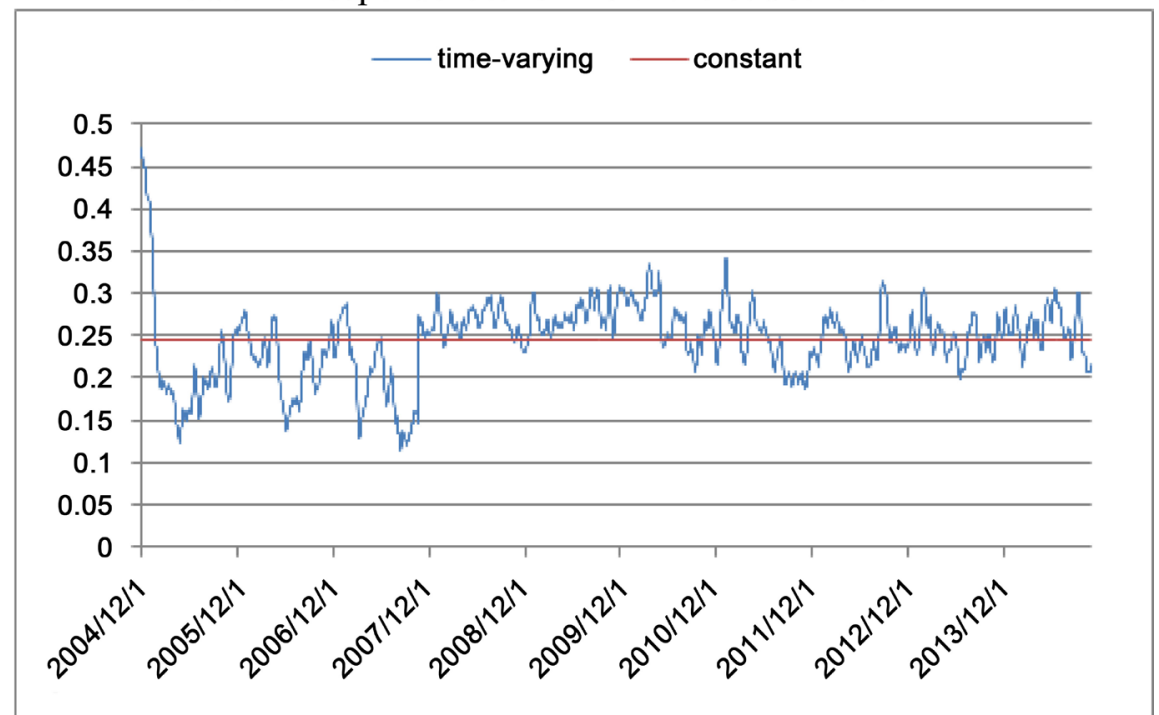

This figure shows the patterns of the upper and lower tail dependences of the time-varying SJC copula and static SJC copula. For the portfolio containing CDX.NA.IG and S \& P500, the lower tail dependence is higher than the upper tail dependence, which implies that the probability of CDX.NA.IG decreasing as S \& P500 increases is higher than that of CDX.NA.IG increasing as S \& P500 decreases.

Figure 2. Upper and lower tail dependences of tvSJC-copula-GARCH-skt.for the portfolio containing CDX.NA.IG and S \& P500. 
Upper tail dependence: CDX.NA.IG \& VIX

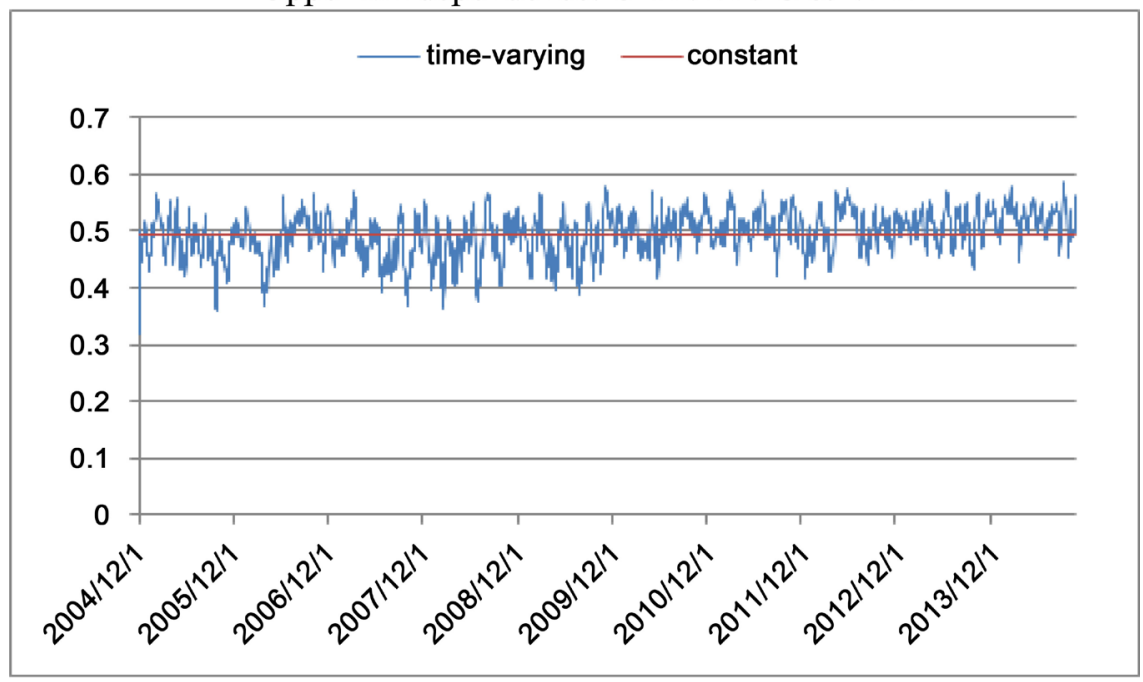

Lower tail dependence: CDX.NA.IG \& VIX

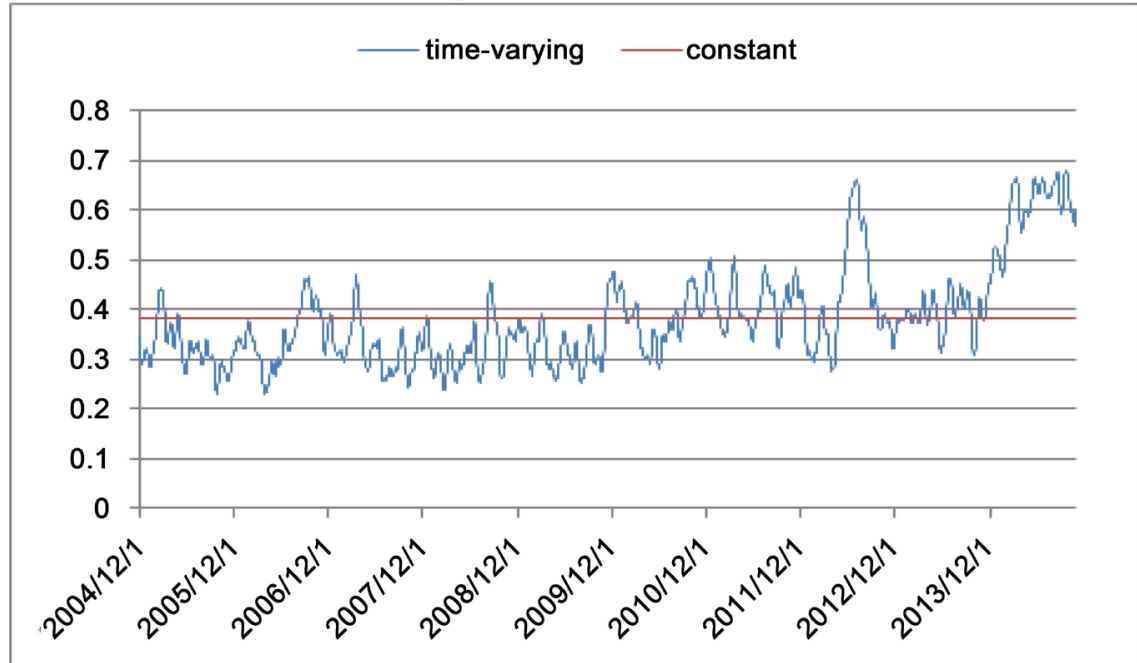

This figure shows the patterns of the upper and lower tail dependences of the time-varying SJC copula and static SJC copula. For the portfolio containing CDX.NA.IG and VIX, the upper tail dependence is higher than the lower tail, which implies that the probability of CDX.NA.IG increasing with VIX is higher than that of CDX.NA.IG decreasing with VIX.

Figure 3. Upper and lower tail dependences of tvSJC-copula-GARCH-skt.for the portfolio containing CDX.NA.IG and VIX.

and the 1-day VaR. Suppose we have 550 in-sample pieces of data. We set $\mathrm{T}$ as $\mathrm{T}=$ 550. By using the rolling window method, when a new observation is added to the data, we remove the oldest one. That is, we use the observations of $t+2$ to $t$ +551 to estimate the $\mathrm{VaR}_{552}$, the observations of $t+3$ to $t+552$ to estimate the $\mathrm{VaR}_{553}$, and so on. The estimated results of the data in the subprime crisis period, the European debt crisis period, and the steady period are shown in Tables 5-7, respectively. Figures 4-6 illustrate the comparisons of 99\% 1-day VaRs with actual portfolio returns in these three individual periods.

Table 5 shows the results of backtesting during the subprime crisis. The subprime crisis, the most eventful period among our three sample periods, gave rise 
Table 5. Numbers of violations of different VaR models. (Subprime crisis period: 2007/02/27-2009/10/02)

\begin{tabular}{|c|c|c|c|}
\hline \multicolumn{4}{|l|}{$\alpha=0.05$} \\
\hline Trading days: 651 & Expected no. of & & 33 \\
\hline \multirow[t]{2}{*}{ Non-rejection region } & \multicolumn{3}{|c|}{$23 \leq$ exceptions $\leq 44$} \\
\hline & CDX.NA.IG S \& P500 & & \\
\hline Historical Simulation & 108 & & \\
\hline Variance-Covariance & 126 & & \\
\hline EWMA & 47 & & \\
\hline GARCH-skt & 56 & & \\
\hline tvSJC-copula-GARCH-skt & $39^{*}$ & & \\
\hline \multicolumn{4}{|l|}{$\alpha=0.01$} \\
\hline Trading days: 651 & \multicolumn{2}{|c|}{ Expected no. of exceptions } & 7 \\
\hline Non-rejection region & \multicolumn{3}{|c|}{$3 \leq$ exceptions $\leq 11$} \\
\hline Historical Simulation & 33 & & \\
\hline Variance-Covariance & 44 & & \\
\hline EWMA & 13 & & \\
\hline GARCH-skt & $9^{*}$ & & \\
\hline tvSJC-copula-GARCH-skt & $5^{*}$ & & \\
\hline
\end{tabular}

This table reports the results of backtesting of the 95\% (99\%) 1-day VaR during the subprime crisis. The historical simulation, the variance-covariance method, EWMA, and the univariate GARCH-skt method are compared with the tvSJC-copula-GARCH-skt, which is the chosen copula-GARCH model with the best goodness-of-fit. Figures with asterisks are in the non-rejection region under 95\% confidence level of LR test.

Table 6. Numbers of violations of different VaR models. (European debt crisis period: 2009/10/05-2012/02/10)

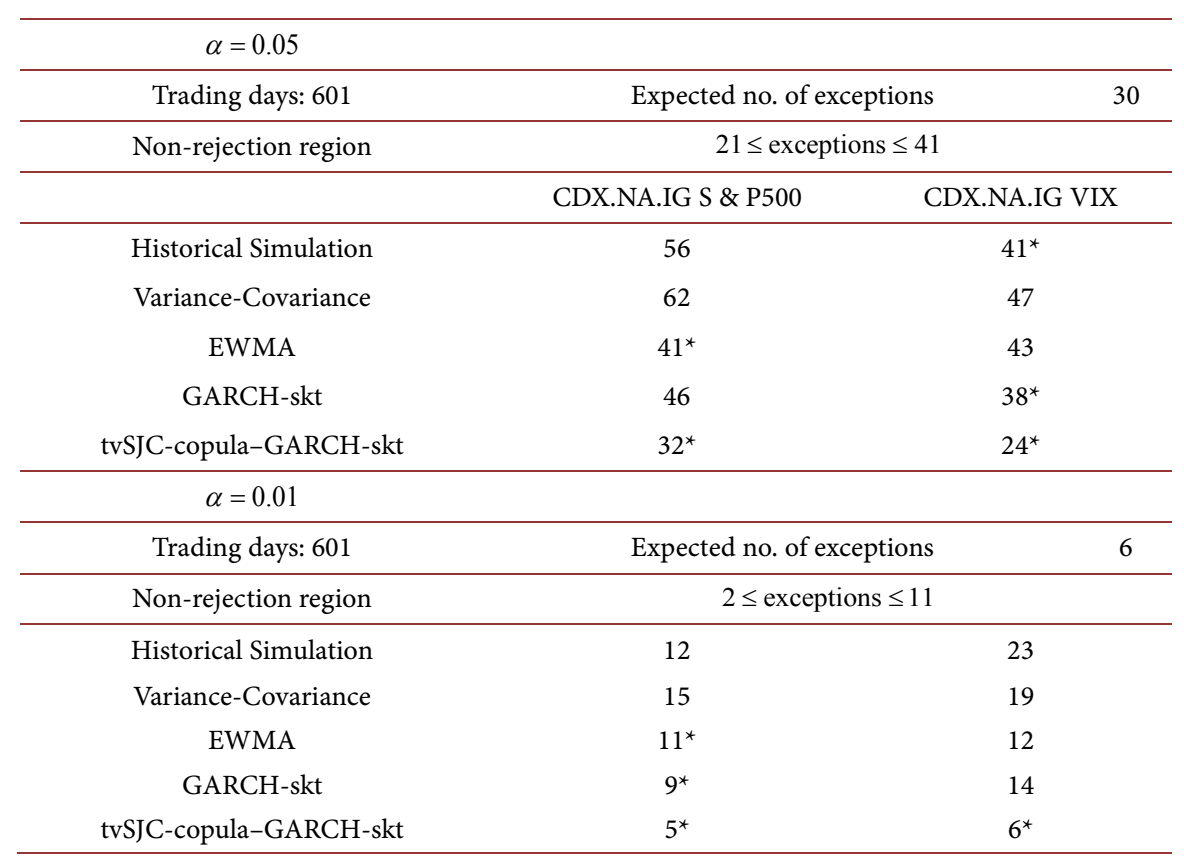

This table reports the results of backtesting of the 95\% (99\%) 1-day VaR during the European debt crisis. The historical simulation, the variance-covariance method, EWMA, and the univariate GARCH-skt method are compared with the tvSJC-copula-GARCH-skt, which is the chosen copula-GARCH model with the best goodness-of-fit. Figures with asterisks are in the non-rejection region under $95 \%$ confidence level of LR test. 
Table 7. Numbers of violations of different VaR models. (Steady period: 2012/02/13 2014/10/31)

\begin{tabular}{|c|c|c|c|}
\hline \multicolumn{4}{|l|}{$\alpha=0.05$} \\
\hline Trading days: 675 & Expected no. of & & 34 \\
\hline \multirow[t]{2}{*}{ Non-rejection region } & \multicolumn{3}{|c|}{$24 \leq$ exceptions $\leq 46$} \\
\hline & CDX.NA.IG S \& P500 & \multicolumn{2}{|c|}{ CDX.NA.IG VIX } \\
\hline Historical Simulation & $45^{*}$ & & \\
\hline Variance-Covariance & 51 & & \\
\hline EWMA & $38^{*}$ & & \\
\hline GARCH-skt & $33^{*}$ & & \\
\hline tvSJC-copula-GARCH-skt & $29^{*}$ & & \\
\hline \multicolumn{4}{|l|}{$\alpha=0.01$} \\
\hline Trading days: 675 & \multicolumn{2}{|c|}{ Expected no. of exceptions } & 7 \\
\hline Non-rejection region & \multicolumn{3}{|c|}{$3 \leq$ exceptions $\leq 12$} \\
\hline Historical Simulation & 21 & & \\
\hline Variance-Covariance & 13 & & \\
\hline EWMA & $12^{*}$ & & \\
\hline GARCH-skt & $12^{*}$ & & \\
\hline tvSJC-copula-GARCH-skt & $8^{*}$ & & \\
\hline
\end{tabular}

This table reports the results of backtesting of the 95\% (99\%) 1-day VaR during the steady period. The historical simulation, the variance-covariance method, EWMA, and the univariate GARCH-skt method are compared with the tvSJC-copula-GARCH-skt, which is the chosen copula-GARCH model with the best goodness-of-fit. Figures with asterisks are in the non-rejection region under $95 \%$ confidence level of LR test.

to significant economic losses and severe worldwide influences. As for the portfolio containing CDX.NA.IG and S \& P500, only tvSJC-copula-GARCH-skt could pass backtesting and estimate accurately under $95 \%$ and $99 \%$ confidence levels. Similar results could be observed for the portfolio containing CDX.NA.IG and VIX. Among the methods, the historical simulation and the variancecovariance method performed the worst, due to their oversimplified assumptions. These two methods tended to provide higher numbers of violations during the subprime crisis, which resulted in underestimating risks for the most eventful period.

The results of backtesting during the European debt crisis are listed in Table 6 . We found that tvSJC-copula-GARCH-skt was still the only method that could pass backtesting and estimate accurately under both $95 \%$ and $99 \%$ confidence levels, no matter which portfolio was under consideration.

Table 7 shows the results of backtesting during the steady period, the most peaceful period among the three. As for the portfolio containing CDX.NA.IG and S \& P500, in addition to tvSJC-copula-GARCH-skt, EWMA and univariate GARCH-skt could also pass backtesting under 95\% and 99\% confidence levels. 
As for the portfolio containing CDX.NA.IG and VIX, every method would pass backtesting under both $95 \%$ and $99 \%$ confidence levels.

The above observations indicate that tvSJC-copula-GARCH-skt is the best method, regardless of period. In contrast, traditional VaR methods fail to give accurate estimations in most cases. Even though EWMA and univariate GARCH-skt are proper estimating methods when the market situation is relatively steady, their estimation ability is weak when markets are volatile, for example, during the subprime crisis and the European debt period. Furthermore, the traditional methods tended to underestimate risk, especially during the subprime crisis period, which was the period when a suitable risk detection method was most needed.

CDX.NA.IG \& S\&P500
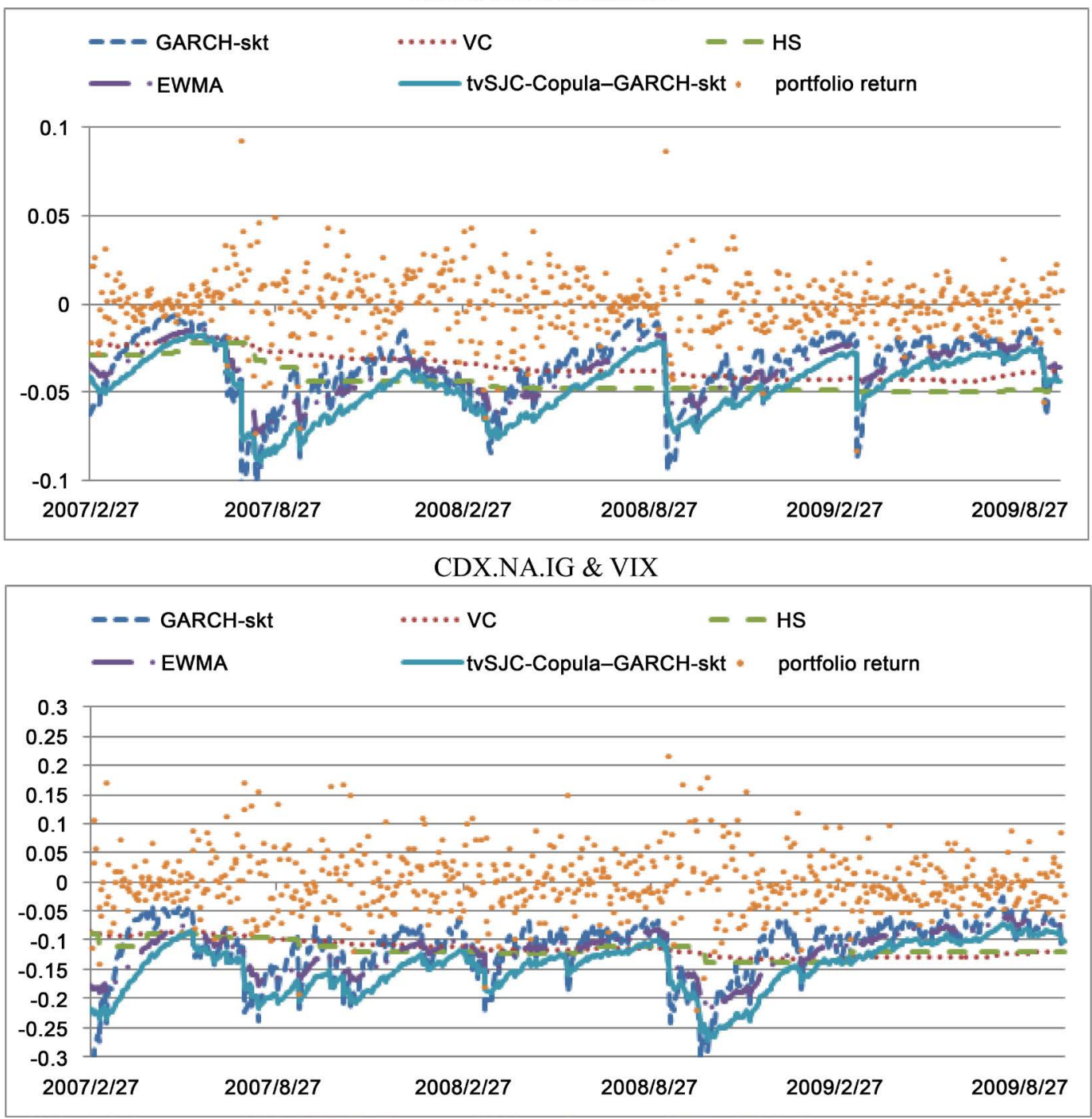

This figure illustrates the results of backtesting of the $99 \%$ 1-day VaR for the two portfolios during the subprime crisis. The historical simulation, the variance-covariance method, EWMA, and the univariate GARCH-skt method are compared with the tvSJC-copula-GARCH-skt, which is the chosen copula-GARCH model with the best goodness-of-fit. The spreading dots are real portfolio return computed using sample date.

Figure 4. Comparison of different VaR models under $\alpha=0.01$ (Subprime crisis period: 2007/02/27-2009/10/02). 

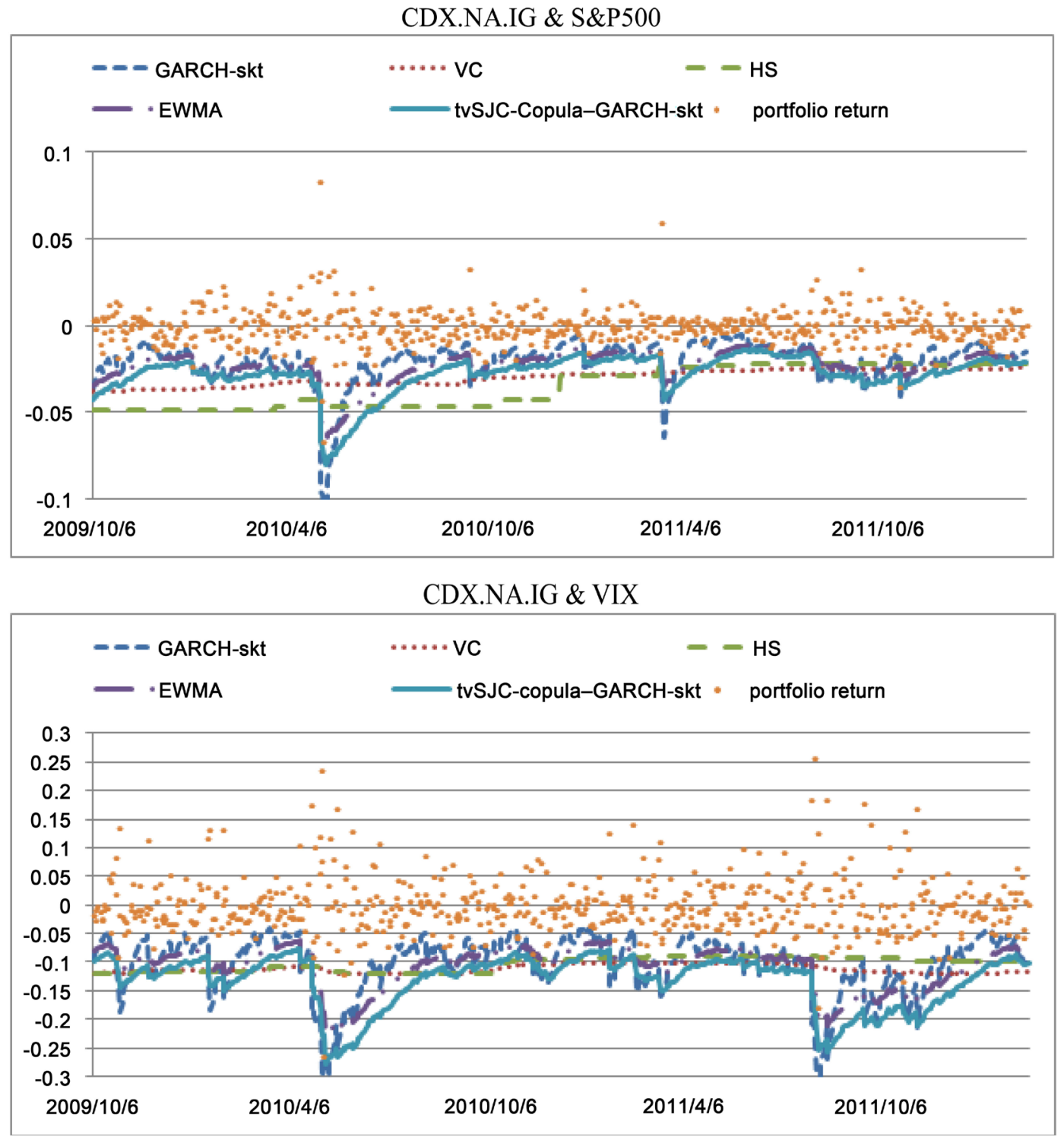

This figure illustrates the results of backtesting of the 99\% 1-day VaR for the two portfolios during the European debt crisis. The historical simulation, the variance-covariance method, EWMA, and the univariate GARCH-skt method are compared with the tvSJC-copula-GARCH-skt, which is the chosen copula-GARCH model with the best goodness-of-fit. The spreading dots are real portfolio return computed using sample date.

Figure 5. Comparison of different VaR models under $\alpha=0.01$ (European debt crisis period: 2009/10/052012/02/10).

Figures 4-6 illustrate the above discussions. The plots of the historical simulation and the variance-covariance method could be treated as strict lines; hence, their reported numbers of violations are far from acceptable in Tables 5-7. By definition, these two methods give equal weight to each past observation, neglecting serial correlations. Although the EWMA and univariate GARCH-skt conquer this problem, they still share the weakness of neglecting the nonlinear correlations between the index returns. They could only show their detection power during the relatively stable period. Not surprisingly, the plots of tvSJC-copula-GARCH-skt are very volatile, remaining adjustable to different 
CDX.NA.IG \& S\&P500
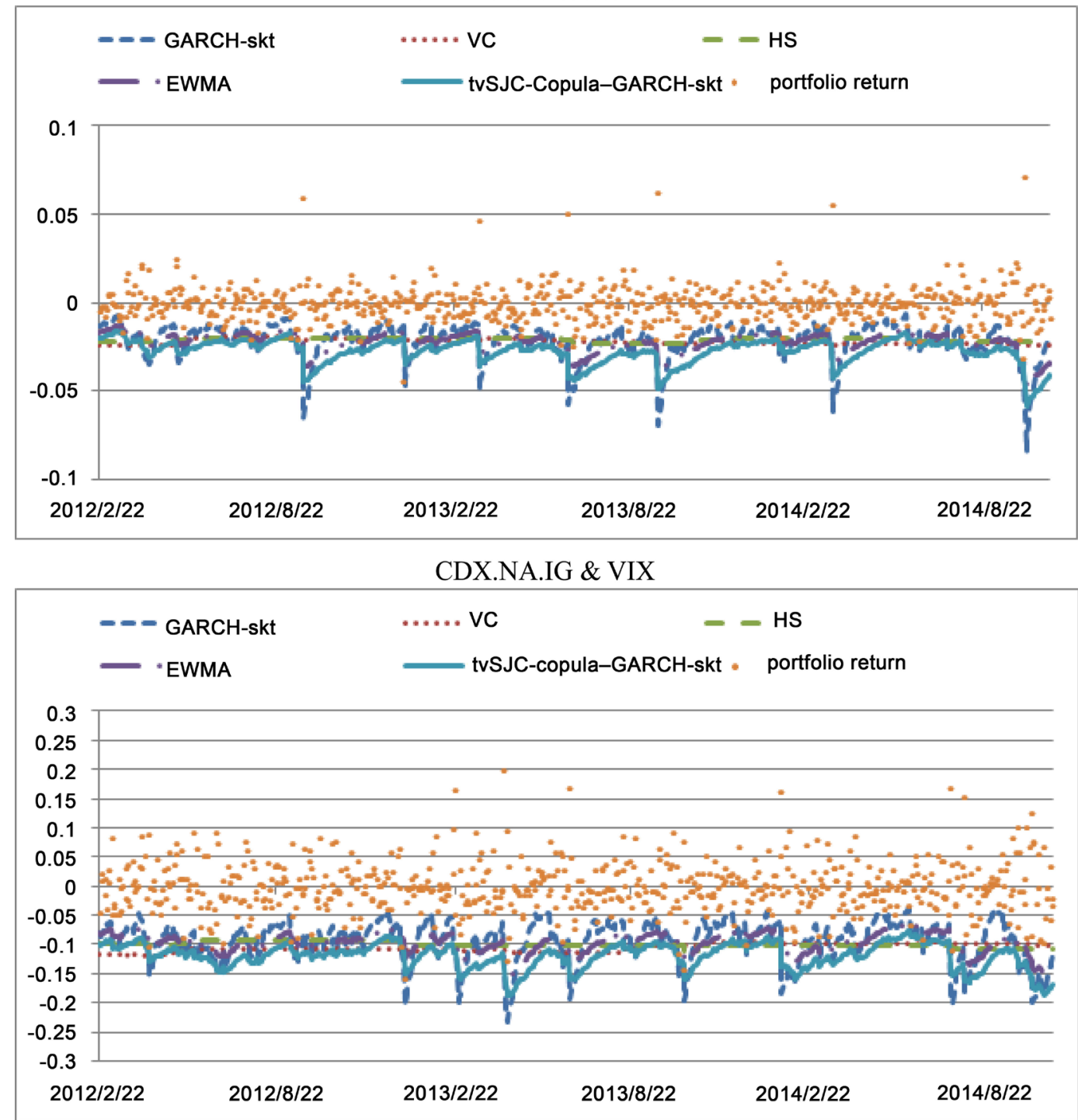

This figure illustrates the results of backtesting of the 99\% 1-day VaR for the two portfolios during the steady period. The historical simulation, the variance-covariance method, EWMA, and the univariate GARCH-skt method are compared with the tvSJC-copula-GARCH-skt, which is the chosen copula-GARCH model with the best goodness-of-fit. The spreading dots are real portfolio return computed using sample date.

Figure 6. Comparison of different VaR models under $\alpha=0.01$ (Steady period: 2012/02/13-2014/10/31).

market situations. The reason why the copula-GARCH methods performed best may be explained not only by their consideration of such features of asset returns as fat tail, asymmetry, and serial correlations, but also by a flexible design dealing with the nonlinear dependence of asset returns. In addition, by comparing these two portfolio, we found that the VaRs for the portfolio containing CDX.NA.IG and VIX are higher than those for the portfolio containing CDX.NA.IG and S \& P 500. That is, the efficiency of risk-diversification is worse in the former case. One possible explanation for this observation could be the correlation coefficient of CDX.NA.IG with VIX is positive, while that with S \& $\mathrm{P}$ is negative. According to the principal of risk diversification, we always obtain 
better shelter when our portfolio containing assets with a negative correlation. In other words, a negative correlation helps to diminish portfolio risk.

\section{Conclusions}

Since they were introduced in the middle of the 1990s, the trading volume of CDSs has soared rapidly, providing an efficient way for commercial banks to diversity away their credit risks. Evidence from previous research has shown that there is a high connection of CDS contracts or indices to the stock or volatility indices. Therefore, for the purpose of risk management, we attempted to find a better way to estimate the VaR of a CDS portfolio with respect to the stock index or volatility index.

The naïve assumption of a linear correlation between the CDS index and the stock or volatility index is no longer suitable to describe their dependence structure. Hence, we need a flexible multivariate distribution with different margins and dependence structures that allows the joint distribution of the portfolio to be free of normality and linear correlation. In addition, such features of the marginal distribution as negative skew, heteroskedasticity, and volatility clustering embedded in financial data should be taken into consideration. In this study, we provided a VaR analysis based on a copula-based GARCH model with a skewed Student-t innovation to improve the detection of rare event risk.

We chose the Markit's North American Investment Grade CDX Index (CDX.NA.IG), which has very high trading volume, as our research object. We constructed two portfolios of the CDX.NA.IG index, one with the S \& P 500 index and the other with VIX. Using the 2477 daily data items from December 2004 to October 2014, covering the period of the subprime mortgage crisis and the European debt crisis, we found that, on average, the VaRs for the portfolio containing CDX.NA.IG and S \& P 500 are lower than those for the portfolio containing CDX.NA.IG and VIX. That is, the efficiency of risk-diversification is better in the former case. In addition, we showed that the time-varying SJC copula model combined with the GARCH with skewed Student-t innovation (tvSJC-copula-GARCH-skt) was superior to the traditional VaR methods and performed best to detect the risk of the portfolios, regardless of the market situation. The advantage of this method mainly results from its consideration of the conditional heteroskedasticity of time series data and its being a flexible way to describe the varying dependence of data structures over time, which conquers such traditional VaR methods as the historical simulation, variance-covariance method, EWMA, and univariate GARCH. Even though the parameters to be estimated are large in number and the computation can be time-wasting, it is worth using the tvSJC-copula-GARCH-skt method to estimate VaR for its relatively correct estimation of rare event risks, even when the market is turbulent.

\section{Acknowledgements}

The authors are very grateful to the Honorary Editor, Michael McAleer and an 
anonymous reviewer for their helpful comments and suggestions, which improved the quality of this paper a lot.

\section{References}

[1] Weistroffer, C., Speyer, B. and Walter, N. (2009) Credit Default Swaps. Deutsche Bank Research.

[2] Blanco, R., Brennan, S. and Marsh, I.W. (2005) An Empirical Analysis of the Dynamic Relation between Investment-Grade Bonds and Credit Default Swaps. Journal of Finance, 60, 2255-2281. https://doi.org/10.1111/j.1540-6261.2005.00798.x

[3] Norden, L. and Weber, M. (2009) The Co-Movement of Credit Default Swap, Bond and Stock Markets: An Empirical Analysis. European Financial Management, 15, 529-562. https://doi.org/10.1111/j.1468-036X.2007.00427.x

[4] Ericsson, J., Jacobs, K. and Oviedo, R. (2009) The Determinants of Credit Default Swap Premia. Journal of Financial and Quantitative Analysis, 44, 109-132. https://doi.org/10.1017/S0022109009090061

[5] Merton, R.C. (1974) On the Pricing of Corporate Debt: The Risk Structure of Interest Rates. Journal of Finance, 29, 449-470.

[6] Zhang, B.Y., Zhou, H. and Zhu, H. (2009) Explaining Credit Default Swap Spreads with the Equity Volatility and Jump Risks of Individual Firms. Review of Financial Studies, 22, 5099-5131. https://doi.org/10.1093/rfs/hhp004

[7] Cao, C., Yu, F. and Zhong, Z. (2010) The Information Content of Option-Implied Volatility for Credit Default Swap Valuation. Journal of Financial Markets, 13, 321-343. https://doi.org/10.1016/j.finmar.2010.01.002

[8] Byström, H. (2005) Credit Default Swaps and Equity Prices: The iTraxx CDS Index Market. Working Papers, Department of Economics, Lund University, Lund.

[9] Alexander, C. and Kaeck, A. (2008) Regime Dependent Determinants of Credit Default Swap Spreads. Journal of Banking \& Finance, 32, 1008-1021. https://doi.org/10.1016/j.jbankfin.2007.08.002

[10] Fung, H., Sierra, G., Yau, J. and Zhang, G. (2008) Are the US Stock Market and Credit Default Swap Market Related? Evidence from the CDX Indices. Journal of Alternative Investments, 11, 43-61. https://doi.org/10.3905/jai.2008.708849

[11] Giammarino, F. and Barrieu, P. (2009) A Semiparametric Model for the Systematic Factors of Portfolio Credit Risk Premia. Journal of Empirical Finance, 16, 655-670. https://doi.org/10.1016/j.jempfin.2009.05.001

[12] Jondeau, E. and Rockinger, M. (2006) The Copula-GARCH Model of Conditional Dependencies: An International Stock Market Application. Journal of International Money and Finance, 25, 827-853. https://doi.org/10.1016/j.jimonfin.2006.04.007

[13] Huang, J.J., Lee, K.J., Liang, H. and Lin, W.F. (2009) Estimating Value at Risk of Portfolio by Conditional Copula-GARCH Method. Insurance: Mathematics and Economics, 45, 315-324. https://doi.org/10.1016/j.insmatheco.2009.09.009

[14] Rong, N. and Trück, S. (2010) Returns of REITS and Stock Markets: Measuring Dependence and Risk. Journal of Property Investment \& Finance, 28, 34-57. https://doi.org/10.1108/14635781011020010

[15] Patton, A.J. (2006) Modelling Asymmetric Exchange Rate Dependence. International Economic Review, 47, 527-556. https://doi.org/10.1111/j.1468-2354.2006.00387.x

[16] So, L. and Yu, J. (2015) Improved Detection of Rare-Event Risk of a Portfolio with 
U.S. REITs. Annals of Financial Economics, 10, Article ID: 1550015. https://doi.org/10.1142/S2010495215500153

[17] McAleer, M. (2014) Asymmetry and Leverage in Conditional Volatility Models. Econometrics, 2, 145-150. https://doi.org/10.3390/econometrics2030145

[18] Hansen, B.E. (1994) Autoregressive Conditional Density Estimation. International Economic Review, 35, 705-730. https://doi.org/10.2307/2527081

[19] Sklar, M. (1959) Fonctions de répartition à n dimensions et leurs marges. Université Paris, Paris, 8.

[20] Gumbel, E.J. (1960) Bivariate Exponential Distributions. Journal of the American Statistical Association, 55, 698-707. https://doi.org/10.1080/01621459.1960.10483368

[21] Clayton, D.G. (1978) A Model for Association in Bivariate Life Tables and Its Application in Epidemiological Studies of Familial Tendency in Chronic Disease Incidence. Biometrika, 65, 141-151. https://doi.org/10.1093/biomet/65.1.141

[22] Frank, M.J. (1979) On the Simultaneous Associativity of F (x, y) and x+y-F (x, y). Aequationes Mathematicae, 19, 194-226. https://doi.org/10.1007/BF02189866

[23] Joe, H. (1997) Multivariate Models and Multivariate Dependence Concepts. CRC Press, Boca Raton. https://doi.org/10.1201/b13150

[24] Longin, F. and Solnik, B. (2001) Extreme Correlation of International Equity Markets. Journal of Finance, 56, 649-676. https://doi.org/10.1111/0022-1082.00340

[25] Okimoto, T. (2008) New Evidence of Asymmetric Dependence Structures in International Equity Markets. Journal of Financial and Quantitative Analysis, 43, 787-815. https://doi.org/10.1017/S0022109000004294

[26] Ang, A. and Chen, J. (2002) Asymmetric Correlations of Equity Portfolios. Journal of Financial Economics, 63, 443-494. https://doi.org/10.1016/S0304-405X(02)00068-5

[27] Fei, F., Fuertes, A. and Kalotychou, E. (2013) Modeling Dependence in CDS and Equity Markets: Dynamic Copula with Markov-Switching. https://papers.ssrn.com/sol3/papers.cfm?abstract_id=2161570

[28] Hull, J. (2012) Options, Futures and Other Derivatives. 8th Edition, Pearson Education, London. 